\title{
Do descriptive norms related to parents and friends predict fruit and vegetable intake similarly among 11-year-old girls and boys?
}

\author{
Elviira Lehto ${ }^{1,2 *}$, Carola Ray ${ }^{1}$, Ari Haukkala ${ }^{3}$, Agneta Yngve ${ }^{4}$, Inga Thorsdottir ${ }^{5}$ and Eva Roos ${ }^{1,2}$ \\ ${ }^{1}$ Folkhälsan Research Center, Topeliuksenkatu 20, O0250 Helsinki, Finland \\ ${ }^{2}$ Department of Public Health, University of Helsinki, OOO14 University of Helsinki, Helsinki, Finland \\ ${ }^{3}$ Department of Social Research, University of Helsinki, O0014 University of Helsinki, Helsinki, Finland \\ ${ }^{4}$ School of Hospitality, Culinary Arts and Meal Science, Örebro University, Campus Grythyttan, 70182 Örebro, Sweden \\ ${ }^{5}$ Unit for Nutrition Research, Landspitali University Hospital, 101 Reykjavik, Iceland E Faculty of Food Science and Nutrition, \\ School of Health Sciences, University of Iceland, 101 Reykjavik, Iceland
}

(Submitted 15 January 2015 - Final revision received 28 August 2015 - Accepted 10 September 2015 - First published online 9 October 2015)

\begin{abstract}
We examined whether there are sex differences in children's fruit and vegetable (FV) intake and in descriptive norms (i.e. perceived FV intake) related to parents and friends. We also studied whether friends' impact is as important as that of parents on children's FV intake. Data from the PRO GREENS project in Finland were obtained from 424 children at the age 11 years at baseline. At baseline, 2009 children filled in a questionnaire about descriptive norms conceptualised as perceived FV intake of their parents and friends. They also filled in a validated FFQ that assessed their FV intake both at baseline and in the follow-up in 2010. The associations were examined with multi-level regression analyses with multi-group comparisons. Girls reported higher perceived FV intake of friends and higher own fruit intake at baseline, compared with boys, and higher vegetable intake both at baseline and in the follow-up. Perceived FV intake of parents and friends was positively associated with both girls' and boys' FV intake in both study years. The impact of perceived fruit intake of the mother was stronger among boys. The change in children's FV intake was affected only by perceived FV intake of father and friends. No large sex differences in descriptive norms were found, but the impact of friends on children's FV intake can generally be considered as important as that of parents. Future interventions could benefit from taking into account friends' impact as role models on children's FV intake.
\end{abstract}

Key words: Fruit and vegetable intake: Descriptive norms: Parents: Friends: Children: Sex differences

Adequate fruit and vegetable (FV) intake has been shown to prevent many common health problems such as being overweight $^{(1)}$, type II diabetes ${ }^{(2)}, \mathrm{CVD}^{(3)}$ and some types of cancer $^{(4)}$. According to the recommendations of the World Health Organization $^{(5)}$, one should eat at least $400 \mathrm{~g}$ of $\mathrm{FV} / \mathrm{d}$. Although the recommendation is directed at adults, for 11-year-old children the aim can be set near to the recommended amount. The actual daily FV intake among Finnish children, however, is $<240 \mathrm{~g}^{(6)}$, and many children do not eat $\mathrm{FV}$ daily at all ${ }^{(7,8)}$. As the consumption pattern of $\mathrm{FV}$ in childhood can be tracked into adulthood $^{(9,10)}$, it is important to understand the factors that predispose children's FV intake to become a habit. Boys have generally been noted to eat less FV than girls ${ }^{(11)}$. Such sex differences in FV intake among Finnish children have not been found to be present in early childhood but to develop during elementary school, which causes energy-adjusted FV intake to be lower among boys than among girls ${ }^{(12)}$. In an international report ${ }^{(13)}$, the growth of sex differences in fruit intake among
Finnish children was reported to occur at the onset of adolescence.

As suggested by Bandura ${ }^{(14)}$ in his social cognitive theory, perceiving someone to behave in a certain way influences one's own behaviour. It is also more likely that a person will follow the example of the other person if this other person is seen as similar to oneself ${ }^{(14)}$. Perceiving a certain behaviour can also be understood as a descriptive norm, as it merely describes how one thinks that other people behave. Perceived parental FV intake has been identified as one of the strongest associates of FV intake among school-aged children in diverse reviews ${ }^{(11,15,16)}$. The importance of parental behaviour, however, can vary by sex. Parental FV intake has been reported, for example, to correlate only with girls' FV intake $^{(17)}$. Among 9-11-year-old Finnish children, FV intake of mothers correlated positively with FV intake among both girls and boys, whereas fathers' FV intake was associated only with boys' FV intake ${ }^{(12)}$.

Abbreviation: FV, fruit and vegetable.

* Corresponding author: E. Lehto, fax +358 93155102, email elviira.lehto@folkhalsan.fi 
A recent review ${ }^{(18)}$ states that children's eating behaviour is also strongly influenced by the eating habits of their peers and friends. Schoolchildren spend much time with their peers and friends, and in Finland they also share daily meals at school. Distinction between friends and peers can be made as describing peers to be persons of similar age and/or developmental level as the child but not acquainted to the child to the same level of intimacy that friends or family members are. The impact of peers on children's FV intake has often been studied in experimental settings, and surveys that examine the impact of friends on children's FV intake have yielded somewhat contradictory results. Perceived FV intake of friends has been positively associated with FV intake among 11- and 12-year-old children in a study by Vereecken et al. ${ }^{(19)}$. Another study ${ }^{(20)}$ in elementary schoolchildren showed a positive association only between perceived FV intake of friends and children's objectively measured fruit intake. On the contrary, with the subjective self-reporting of fruit intake or vegetable intake, no association of perceived $\mathrm{FV}$ intake of friends was found ${ }^{(20)}$. In adolescents, however, best friends' dietary behaviour was associated among others with vegetable intake but not with fruit intake $^{(21)}$. In a longitudinal study setting, one's best friend's healthy eating (which was defined as eating FV in most days and limiting one's intake of junk food) did not predict adolescents' FV intake 2 years later ${ }^{(22)}$. An earlier study that examined the effect of parents' and friends' FV intake as a single variable found that descriptive norm only had an impact on girls' FV intake ${ }^{(23)}$

Sex differences in FV intake have often been noted, but the sex aspect in understanding the determinants of $\mathrm{FV}$ intake has been taken into account in only few studies. Girls have reported, particularly in some Nordic countries, higher levels of social determinants (e.g. parental encouragement and demand) that are associated with $\mathrm{FV}$ intake ${ }^{(24)}$. In addition, in an Icelandic study, perceived FV intake of parents and friends was found to be higher among girls, and it also was associated with FV intake among girls but not among boys ${ }^{(23)}$. Sex differences in FV intake in a study by Bere et al. ${ }^{(25)}$ were mainly explained by differences in taste preferences, but perceived FV intake of parents, friends, siblings and teacher also acted as a mediator. To the best of our knowledge, no studies with longitudinal settings and focus on sex differences in perceived FV intake of friends and parents have been conducted.

As shown in the above-mentioned studies, descriptive norms can be considered important for the formation of children's eating behaviour. As fruit intake and vegetable intake can be regarded as somewhat different kinds of behaviours, which have different predictors or barriers ${ }^{(26,27)}$, we wanted to study the associations separately for fruit intake and for vegetable intake. In the present study, we examined whether there are sex differences in children's self-reported fruit or vegetable intake and whether those are affected by their perception of FV intake of parents and friends. We hypothesised that among 11-yearold schoolchildren the impact of friends, in addition to parental influence, would be strong. It is possible that these children regard friends as being more similar to them than their own parents and therefore more valued as role models. We also wanted to examine whether descriptive norms are gendered in a way that girls would be more influenced by their mothers, and fathers would have more impact upon their sons' FV intake.

\section{Methods}

\section{Study design and participants}

The PRO GREENS project (www.progreens.org) has focused on the promotion of FV intake among 11-year-old children in ten European countries in 2009 and 2010. The data that were used in this study were obtained from a regional sample of Swedishspeaking schools in the coastal regions of Finland. As the data were originally collected for an international intervention study, the aim was to recruit 1000 children in order to ensure a sufficient level of statistical power. According to the power calculation (http://webpower.psychstat.org/models/ reg01/), which we conducted afterwards for the current study including only the children in the control schools, the required sample size for the most complex models was 236 if the power level was set at $0 \cdot 8$. An invitation to participate was mailed to the schools, and in some cases it was followed up by a reminder phone call. All invited schools were willing to participate and were randomised into intervention and control schools. Only the sample from the control schools ( $n$ 553) was included in the analyses of the present study. Baseline took place in May 2009 when descriptive norms were assessed. A human error at carrying out the translation task of the questionnaires led to the exclusion of one side of the questionnaire (which assessed children's FV intake) in May 2009. Therefore, the baseline measurements for children's FV intake were conducted in August and September 2009. The follow-up took place in May 2010, when children reported both their FV intake and filled in the questionnaire for assessing descriptive norms. Children filled in the paper questionnaires in the classrooms under the guidance of their teacher. A consent form and a questionnaire assessing the educational levels of their parents were brought home by the children. In May 2009, 492 children (response rate $89 \%$ ), who were in the fourth and fifth grade, participated in the study. Those children whose response from all time points was received ( $n$ 424, $77 \%$ from the original sample) and whose parents returned the consent form are included in the analyses. Most dropouts were because of moving to another area and because of a change of school. The study protocol was approved by the Ethics Committee of the Department of Public Health in the University of Helsinki, and the parents provided their informed written consent for their children.

\section{Measures}

FV intake of the children was measured by filling in a validated FFQ, which was developed for the Pro Children study ${ }^{(28)}$. Questions about the frequency of fresh fruit intake, salad intake, raw vegetable intake and cooked vegetable intake were asked separately. Answer alternatives were as follows: (1) never; (2) less than once a week; (3) once a week; (4) 2-4 d/week; (5) 5-6 d/week; (6) every day, once a day; (7) every day, twice a day; and (8) every day, more than twice a day. Answers were reweighed to describe the times per $d$ a child eats 
fruits/vegetables. Intakes of salad, raw vegetables and cooked vegetables were added together to a variable indicating vegetable intake.

Descriptive norms/perceived fruit or vegetable intake of mother, father and friends were defined by responding to statements 'My mother/father/best friends eat(s) fruit/ vegetables every day'. Questions were adopted from the validated questionnaire of the Pro Children study ${ }^{(29)}$ and asked separately for fruit intake and for vegetable intake. Answer alternatives were as follows: (1) I fully agree, (2) I agree somewhat, (3) I don't agree nor disagree, (4) I disagree somewhat and (5) I fully disagree. Questions about perceived FV intake of mother or father had also an answer alternative: (6) I don't have a/meet mother/father. Children who did not answer the question or chose the sixth option ( $n$ 2-16, depending on the sex of the parent and time) were excluded from the analyses. The answers were recoded so that a higher digit indicates a higher level of descriptive norms (i.e. increased fruit or vegetable intake of parents or friends).

Parental educational level was enquired from the respondent parent, who was asked to select the highest educational level that she/he had attained from the following: (1) not completed elementary school, (2) elementary school, (3) high school, (4) senior high school, (5) college or university or (6) other education. This variable was used in the analyses as a continuous adjustment variable.

\section{Statistical methods}

All analyses were conducted separately for fruit intake and vegetable intake. Bivariate associations between descriptive norms and children's FV intake were assessed by Spearman's correlation coefficients as run on SPSS Statistics 20.0 for Windows. Linear regression analyses were used to study how descriptive norms at baseline affected children's fruit intake and vegetable intake at baseline and in the follow-up. As we obtained high correlations between descriptive norm variables, we examined the association between each descriptive norm variable and FV intake separately. These results are presented in the tables. To confirm that the results are concise, we also ran analyses in which all descriptive norm variables (perceived FV intake of mother, father and friends) were included in the models simultaneously. The results of these analyses are presented only in the text. All analyses were adjusted for children's sex (when girls and boys were analysed together), age and parental educational level. Possible differences between girls and boys in the studied associations were examined by using multi-group analyses. Significance level was set at $P<0 \cdot 1$ for multi-group analyses $^{(30)}$ and at $P<0.05$ for all other analyses. To take into account the nesting in school classes in the sampling, we conducted all regression analyses by using the Complex command in Mplus statistical software version 7.1 $1^{(31)}$.

\section{Results}

The characteristics of the study sample in total and also by sex are presented in Table 1. At baseline, girls were eating fruit more often than boys, but in the follow-up no sex differences in fruit intake were found. Vegetable intake was higher among girls than among boys at both time points. The sex difference in the change in fruit or vegetable intake was not statistically significant. At baseline, girls reported higher perceived fruit intake of friends more often than boys did. In other descriptive norm variables, there were no sex differences found. High positive correlations were found between perceived fruit intake of mother and perceived fruit intake of father $(r 0.42 ; P<0.001)$, as well as between perceived vegetable intake of mother and perceived vegetable intake of father $(r 0.65 ; P<0 \cdot 001)$ (data not shown).

\section{Fruit intake}

Table 2 presents the associations from linear regression analyses between perceived fruit intake of mother, father and

Table 1. Sample characteristics in total sample and by sex

(Number and percentage; mean values and standard deviations)

\begin{tabular}{|c|c|c|c|c|c|c|c|}
\hline & \multicolumn{2}{|c|}{ Total } & \multicolumn{2}{|c|}{ Girls } & \multicolumn{2}{|c|}{ Boys } & \multirow[b]{2}{*}{$P^{*}$} \\
\hline & Mean & SD & Mean & SD & Mean & SD & \\
\hline$n$ & \multirow{2}{*}{\multicolumn{2}{|c|}{424}} & \multirow{2}{*}{\multicolumn{2}{|c|}{$\begin{array}{r}205 \\
48\end{array}$}} & \multicolumn{2}{|c|}{219} & \\
\hline$\%$ & & & & & & & \\
\hline Age at baseline (years) & 11.4 & 0.6 & $11 \cdot 3$ & 0.6 & 11.4 & 0.6 & 0.332 \\
\hline Fruit intake at baseline (times/d) & 0.87 & 0.75 & 0.97 & 0.81 & 0.78 & 0.67 & 0.009 \\
\hline Fruit intake at follow-up (times/d) & 0.85 & 0.72 & 0.92 & 0.74 & 0.79 & 0.70 & 0.062 \\
\hline Fruit intake (change) & -0.02 & 0.77 & -0.06 & 0.83 & 0.01 & 0.70 & 0.374 \\
\hline Vegetable intake at baseline (times/d) & 1.66 & $1 \cdot 18$ & 1.91 & 1.28 & 1.42 & 1.03 & $<0.001$ \\
\hline Vegetable intake at follow-up (times/d) & 1.57 & 1.12 & 1.79 & $1 \cdot 10$ & 1.37 & $1 \cdot 11$ & $<0.001$ \\
\hline Vegetable intake (change) & -0.09 & $1 \cdot 10$ & -0.13 & $1 \cdot 17$ & -0.06 & 1.03 & 0.523 \\
\hline Perceived fruit intake of mother & 3.9 & 0.89 & 4.0 & 0.87 & 3.8 & 0.91 & 0.118 \\
\hline Perceived fruit intake of father & 3.4 & 1.05 & $3 \cdot 3$ & 1.11 & $3 \cdot 4$ & 1.00 & 0.377 \\
\hline Perceived fruit intake of friends & 3.5 & 0.86 & $3 \cdot 6$ & 0.82 & 3.4 & 0.90 & 0.025 \\
\hline Perceived vegetable intake of mother & $4 \cdot 1$ & 0.94 & 4.2 & 0.95 & $4 \cdot 1$ & 0.92 & 0.752 \\
\hline Perceived vegetable intake of father & $3 \cdot 7$ & $1 \cdot 12$ & 3.7 & 1.09 & 3.8 & $1 \cdot 15$ & 0.426 \\
\hline Perceived vegetable intake of friends & 3.5 & 0.90 & 3.5 & 0.90 & 3.4 & 0.89 & 0.305 \\
\hline
\end{tabular}

* Difference in the means between girls and boys. 
Descriptive norms, fruit and vegetable intake

friends at baseline and children's own fruit intake at baseline and in the follow-up and the change in fruit intake from baseline to the follow-up. Perceiving higher fruit intake of mother, father and friends all had a positive impact on children's fruit intake at baseline and in the follow-up. Only perceived fruit intake of one's father and friends contributed to the change in children's fruit intake. The only statistically significant difference between sexes according to multi-group analyses was the associations between perceived fruit intake of mother at baseline and children's fruit intake at baseline. This association was found to be stronger among boys, and because of the significant interaction effect we show the results for perceived fruit intake of the mother separately for girls and for boys.

We also tested models that included all three descriptive norm variables simultaneously (data not shown). The results were parallel to the separate analyses, but the associations were weaker than in the single regression models. Baseline fruit intake was associated with perceived fruit intake of mother $(\beta 0.22 ; 95 \%$ CI $0.11,0.33 ; P<0.001)$ and friends $(\beta 0.13 ; 95 \%$ CI $0.01,0.25 ; P<0.001)$ but not with perceived fruit intake of father $(\beta 0.07 ; 95 \% \mathrm{CI}-0 \cdot 03,0 \cdot 18)$. When predicting fruit intake in the follow-up or the change in fruit intake from baseline to follow-up, both perceived fruit intakes of father $(\beta 0 \cdot 19 ; 95 \% \mathrm{CI}$ $0.09,0.30$ and $\beta 0 \cdot 17 ; 95 \%$ CI $0.07,0 \cdot 27$, respectively; both $P<0.01)$ and friends $(\beta 0.17 ; 95 \%$ CI $0.08,0.23$ and $\beta 0.11 ; 95 \%$ CI $0 \cdot 03,0 \cdot 19$, respectively; both $P<0 \cdot 01)$ were significant predictors, but the effect of mother was non-significant $(\beta 0.03$; $95 \%$ CI $-0.09, \quad 0.15$ and $\beta-0.06 ; 95 \%$ CI $-0.17,0.03$, respectively).

\section{Vegetable intake}

The impact of perceived vegetable intake of mother, father and friends on children's vegetable intake is shown in Table 3. Perceived vegetable intakes of mother, father and friends at baseline were all positively associated with children's vegetable intake at baseline and in the follow-up. The impact of mother became non-significant when predicting the change in children's vegetable intake. The pattern of the effect of perceived vegetable intake of parents and friends on vegetable intake was similar among girls and boys in the multi-group analyses.

As with fruit intake, we also ran analyses that included all descriptive norm variables in the models simultaneously (data not shown). When perceived vegetable intakes of mother, father and friends were examined together, none of them were significantly associated with children's vegetable intake at baseline ( $\beta$ 0.03; 95\% CI $-0.10,0.15, \beta 0.14 ; 95 \% \mathrm{CI}-0.01,0.30$ and $\beta 0.10 ; 95 \%$ CI $-0.02,0 \cdot 23$, respectively). When predicting vegetable intake in the follow-up, both perceived vegetable intakes of father $(\beta 0 \cdot 22 ; 95 \% \mathrm{CI} 0 \cdot 11,0 \cdot 34 ; P<0 \cdot 001)$ and friends ( $\beta 0.15 ; 95 \%$ CI $0.05,0.25 ; P<0.01$ ) were significant predictors, whereas the effect of perceived vegetable intake of mother was non-significant $(\beta-0.06 ; 95 \% \mathrm{CI}-0 \cdot 18,0.06)$. The change in children's vegetable intake was affected only by perceived vegetable intake of father $(\beta 0.17 ; 95 \% \mathrm{CI} 0.05,0.28 ; P<0.01)$, whereas the effect of mother $(\beta-0 \cdot 08 ; 95 \% \mathrm{CI}-0 \cdot 19,0 \cdot 03)$ and friends $(\beta 0 \cdot 10 ; 95 \% \mathrm{CI}-0 \cdot 00,0 \cdot 20)$ was non-significant. 
Table 3. Linear regression analyses for predicting children's vegetable intake at baseline and follow-up by perceived vegetable intake of mother, father or friends at baseline†

(Regression coefficients, 95\% confidence intervals and coefficients of determination)

\begin{tabular}{|c|c|c|c|c|c|c|c|c|c|c|c|c|}
\hline & \multicolumn{12}{|c|}{ Children's vegetable intake } \\
\hline & \multicolumn{4}{|c|}{ Baseline } & \multicolumn{4}{|c|}{ Follow-up } & \multicolumn{4}{|c|}{ Change } \\
\hline & $n$ & $\beta$ & $95 \% \mathrm{Cl}$ & $R^{2}$ & $n$ & $\beta$ & $95 \% \mathrm{Cl}$ & $R^{2}$ & $n$ & $\beta$ & $95 \% \mathrm{Cl}$ & $R^{2}$ \\
\hline Vegetable intake of mother & 391 & $0 \cdot 12^{*}$ & $0.02,0.23$ & $0 \cdot 10^{\star \star \star}$ & 390 & $0.13^{*}$ & $0.03,0.22$ & $0.13^{\star \star \star}$ & 382 & 0.06 & $-0.02,0.15$ & $0.36^{* * *}$ \\
\hline Vegetable intake of father & 381 & $0 \cdot 19^{* *}$ & $0.07,0.30$ & $0.12^{\star \star}$ & 382 & $0.22^{\star \star \star}$ & $0.14,0.31$ & $0.16^{\star \star \star}$ & 374 & $0 \cdot 14^{\star *}$ & $0.05,0.22$ & $0.38^{\star * *}$ \\
\hline Vegetable intake of friends & 392 & $0.14^{*}$ & $0.01,0.27$ & $0 \cdot 10^{\star *}$ & 390 & $0.18^{\star \star \star}$ & $0.07,0.28$ & $0.14^{\star \star \star}$ & 382 & $0 \cdot 11^{*}$ & $0.01,0.20$ & $0.36^{\star * *}$ \\
\hline
\end{tabular}

${ }^{*} P<0.05,{ }^{* *} P<0.01,{ }^{* * *} P<0.001$

$\dagger$ All descriptive norm factors are studied in separate models. All models are adjusted for sex, age and educational level of the respondent parent.

\section{Discussion}

The aim of the present study was to examine whether there are sex differences in children's FV intake and whether the impact of perceived FV intake of parents and friends is similar on FV intake among both sexes. Girls reported higher levels of fruit intake at baseline and higher vegetable intake both at baseline and in the follow-up. Girls also perceived their friends' fruit intake to be higher than boys did. Higher perceived FV intake of mother, father and friends predicted children's higher FV intake at baseline and in the follow-up. Perceived FV intake of father and friends also predicted an increase in children's (boys and girls) FV intake from baseline to follow-up. The only sex difference was found in the impact of perceived fruit intake of mother on children's own fruit intake, which was stronger among boys than among girls. Friends' influence on children's FV intake was generally as strong as the impact of the parents.

It has been reported that boys tend to eat less FV than girls ${ }^{(11)}$. In the present study among 11-year-old children, such sex differences were also apparent for fruit intake at baseline and for vegetable intake both at baseline and in the follow-up. It should be noted that FV intake was measured as self-reported times per $d$ in this study, which makes the comparison with other Finnish studies somewhat difficult. Girls have previously been found to have the following: higher intake frequency of raw vegetables but not of cooked vegetables or fruits ${ }^{(32)}$, higher daily mean intake of fruits but not of vegetables ${ }^{(6)}$ or both higher fruit intake and vegetable intake when measured as energy-adjusted mean intake ${ }^{(12)}$. As boys receive more energy from their food ${ }^{(33)}$, comparing energy-adjusted mean values of $\mathrm{FV}$ intake could emphasise the small portion of FV in boys' diets. In our study, fruit intake among girls decreased during the follow-up, whereas fruit intake among boys did not change. It is possible that a longer follow-up would reveal another kind of pattern. On the basis of our results, however, it is difficult to determine whether girls are just starting to decrease their fruit intake earlier than boys do or whether it is a slow societal trend that the sex differences in fruit intake are indeed getting smaller or vanishing.

The strongest sex difference was found in the children's perception of fruit intake among friends, which was rated to be higher among girls. Sex differences have been noted to increase in early adolescence for such predictors of $\mathrm{FV}$ intake, as preferences and intention ${ }^{(25)}$ and this kind of development can be assumed to take place with other associates of FV intake as well. It is possible that girls and boys perceive and interpret the environment slightly differently, but an alternative plausible interpretation is that the environment for girls and boys can indeed differ. This could have been the case with the availability of fruits at home, which was reported to be higher by girls and their parents than by boys and their parents ${ }^{(34)}$. Higher scores in perceived fruit intake of friends among girls in our study can reflect girls' higher probability of paying attention to fruit intake of their friends, which could be intertwined with girls' higher appreciation of such eating behaviour. Alternatively, it could simply be that girls' best friends are more likely to be other girls and the perception of their higher fruit intake, compared with boys, is accurate. However, girls did not rate vegetable intake of their friends to be higher at baseline, even though the sex differences in vegetable intake were already present at that time point.

An unexpected result was the relatively low impact of perceived FV intake of the mother, especially among girls and in the follow-up setting, albeit the lack of mother's impact has also been found in Iceland ${ }^{(34)}$. One reason that could explain our finding is the relatively small diversity in mothers' FV intake: between 70 and $75 \%$ of children agreed fully or somewhat with the statements that their mother eats fruits or vegetables daily. In another Finnish study conducted among 9-11-year-olds, mothers' FV intake correlated with FV intake of both girls and boys, whereas fathers' FV intake was only associated with boys' FV intake ${ }^{(12)}$. Such clear sex-specific parental descriptive norm impacts were not found in this sample, which might reflect the differences in the method of measuring between the two studies - that is food records of parents and children in the study of Talvia et $a l{ }^{(12)} v$. self-report questionnaires filled-in only by children in our study. Still, the finding of the father's impact being a strong associate and predictor of children's FV intake even though no maternal effect was found supports our decision to examine the descriptive norm variables separately. Also the interpretation of the models in which all descriptive norm variables were examined simultaneously could be misleading, as the predictor with the lowest effect becomes non-significant partly because of high intercorrelations. However, the fact that FV intake of fathers can be even lower than that of their children ${ }^{(35)}$ is a matter of 
concern, as it can hinder children from adopting healthy eating habits at home.

The positive impact of perceived FV intake of friends upon children's FV intake was generally found to be as strong as that of parents, and no clear pattern of sex differences was noted across the models. Moreover, descriptive norms related to friends seemed to be an important associate and predictor for girls and boys both in cross-sectional and longitudinal settings. In a recent Danish study conducted among 12-year-olds, both perceived FV intake of parents and friends correlated with children's FV intake ${ }^{(36)}$. When included in the same model, however, parental descriptive norm was a stronger associate of children's FV intake, and the correlation between perceived FV intake of friends and children's own FV intake became non-significant ${ }^{(36)}$. The differences of our results could be because of the dissimilarities in the school lunch policy, which in Denmark might give parents more influence on their children's FV intake. On the other hand, in our study, the importance of friends was high especially in the longitudinal setting, which was not included in the Danish study. Previously, descriptive norms have been reported to be strong predictors of adolescents' eating behaviour ${ }^{(37)}$. We noted that already among children in the elementary school friends seem to have an impact on each other's FV intake. The strong impact of best friends implies that children's whole social environment, not only the family context, should be taken into account when aiming at increasing children's FV intake.

Even though perceiving high FV intake among friends can increase children's FV intake, adjusting one's behaviour does not necessarily have to follow the actual behaviour of others. In an experimental study ${ }^{(37)}$, adolescents increased their fruit intake when they were told that their school student peers try to eat a sufficient amount of fruits. Receiving information about such a descriptive norm, without observing the actual behaviour to which it referred, was more efficient than receiving information about the health benefits of fruit consumption or passing on a prescriptive norm that elaborates what others think that you should $\mathrm{do}^{(37)}$. When children grow older, they can become more resistant against attempts to try to change their behaviour, as they consider it to work against their independence. Therefore, perceiving a certain (desired) behaviour or passing the same information verbally could prove to be more efficient in future interventions that aim to increase children's FV intake compared with the more traditional health education, which is prescriptive. Social cognitive theory suggests that a person will more likely be imitated if (s)he is close to the subject or (s)he is otherwise valued by the subject ${ }^{(14)}$. As it is likely to be hard to increase FV intake in a group of friends in which the children are simultaneously observing each other's low FV intake, more distant peers with high FV intake could act as the surrogate role models. Indeed, that was the case in an intervention conducted by Horne et al. ${ }^{(38)}$, in which the imaginary, but heroic, peers (viewed to consume FV on the videos) successfully increased children's FV intake, whereas simply increasing the availability of FV did not result in any changes in $\mathrm{FV}$ intake. In the context of observing real people, it is obvious that to be able to perceive high FV intake of someone, there has to be FV available to consume at least for the person acting as a role model. Therefore, at homes in which children report higher levels of perceived parental FV intake, the availability of FV can also be higher so that the effects of descriptive norms and availability are intertwined. However, it is probable that in the case of friends for whom in Finland the same amount of FV is provided at school lunch the availability does not have such an impact on descriptive norms.

The strengths of the present study are the examination of the different factors of descriptive norms in a follow-up setting and its high participation rate. The use of validated questionnaires, which have been used in many other studies and among different populations, enable the comparison of our results. The main limitation of the study is that the sample was not representative, and thus the results are not necessarily generalisable to all Finnish children. The relatively small sample size and self-reporting of the examined factors can also be seen as limitations. The sample size, however, was smaller than suggested by the power analysis only in the separate analyses for girls and boys, but in these specific analyses the examined associations reached statistical significance. Although 11-year-old children have been found to be able to estimate their FV intake accurately ${ }^{(39)}$, the assessment of the eating behaviour attributed to other people is not necessarily correct, nor is it similar among girls and boys. However, children's subjective perception of parental FV intake has been suggested to be more consistently associated with their own FV intake, compared with when parental FV intake was reported by the parents themselves ${ }^{(15)}$, although the parental report also correlates with children's FV intake in some studies ${ }^{(36,40)}$. Acknowledging the impact of friends on children's FV intake, it would be beneficial to know whether there are differences in the association between children's FV intake and friends' FV intake measured subjectively and objectively.

\section{Conclusions}

We found modest sex differences in children's FV intake and in some of the descriptive norms that predict FV intake. Studies have shown that sex differences in FV intake and its predictors start to occur in late childhood. Among both 11-year-old girls and boys, the impact of friends on FV intake is as strong as that of their parents, which indicates that children's broader social environment should be taken into account when planning future interventions.

\section{Acknowledgements}

The authors would like to express special thanks to all the teachers and children who participated in this survey, and to all the staff and students who contributed to the data collection and entries.

The PRO GREENS project has been made possible through financial support from the European Commission's Programme of Community Action in the Field of Public Health 2003-2008. The study does not necessarily reflect the Commission's views and in no way anticipates its future policy in this area. Additional financial support for the Finnish study group was 
provided by the Juho Vainio Foundation and the Finnish Cultural Foundation.

The authors contributed to the manuscript as follows: E. L. formulated the research questions, had the main responsibility for writing the manuscript and carried out the statistical analyses. C. R., E. R., A. Y. and I. T. designed the study, conducted the data collection and with $\mathrm{A}$. H. revised the manuscript. All authors read and approved the final manuscript.

The authors declare that they have no conflicts of interest.

\section{References}

1. Buijsse B, Feskens EJM, Schulze MB, et al. (2009) Fruit and vegetable intakes and subsequent changes in body weight in European populations: results from the project on Diet, Obesity, and Genes (DiOGenes). Am J Clin Nutr 90, 202-209.

2. Montonen J, Järvinen R, Heliövaara M, et al. (2005) Food consumption and the incidence of type II diabetes mellitus. Eur J Clin Nutr 59, 441-448.

3. Hung HC, Joshipura KJ, Jiang R, et al. (2004) Fruit and vegetable intake and risk of major chronic disease. J Natl Cancer Inst 96, 1577-1584.

4. Riboli E \& Norat T (2003) Epidemiologic evidence of the protective effect of fruit and vegetables on cancer risk. Am J Clin Nutr 78, 559S-569S.

5. World Health Organization (2003) Diet, Nutrition and the Prevention of Chronic Diseases. Joint WHO/FAO Expert Consultation. WHO Technical Report Series no. 916. Geneva: WHO.

6. Lynch C, Kristjansdottir AG, Te Velde S, et al. (2014) Fruit and vegetable consumption in a sample of 11-year old children in ten European countries - the PRO GREENS crosssectional survey. Public Health Nutr 17, 2436-2444.

7. Hoppu U, Lehtisalo J, Tapanainen H, et al. (2010) Dietary habits and nutrient intake of Finnish adolescents. Public Health Nutr 13, 965-972.

8. Lehto E, Ray C, Te Velde S, et al. (2015) Mediation of parental educational level on fruit and vegetable intake among schoolchildren in ten European countries. Public Health Nutr 18, 89-99

9. Lien N, Lytle L \& Klepp K (2001) Stability in consumption of fruit, vegetables, and sugary foods in a cohort from age 14 to age 21. Prev Med 33, 217-226.

10. Te Velde S, Twisk JWR \& Brug J (2007) Tracking of fruit and vegetable consumption from adolescence into adulthood and its longitudinal association with overweight. BrJ Nutr $\mathbf{9 8}$, 431-438.

11. Rasmussen M, Krølner R, Klepp K, et al. (2006) Determinants of fruit and vegetable consumption among children and adolescents: a review of the literature. Part I: quantitative studies. Int J Behav Nutr Phys Act 3, 22.

12. Talvia S, Räsänen L, Lagström H, et al. (2006) Longitudinal trends in consumption of vegetables and fruit in Finnish children in an atherosclerosis prevention study (STRIP). Eur J Clin Nutr 60, 172-180.

13. World Health Organization (2008) Inequalities in Young People's Health. HBSC International Report from the 2005/2006 Survey, vol. 5. Geneva: World Health Organization.

14. Bandura A (1986) Social Foundations of Thought and Action. Englewood Cliffs, NJ: Prentice-Hall.

15. McClain AD, Chappuis C, Ngyen-Rodriguez ST, et al. (2009) Psychosocial correlates of eating behavior in children and adolescents: a review. Int J Behav Nutr Phys Act 6, 54.
16. Pearson N, Biddle SJH \& Gorely T (2009) Family correlates of fruit and vegetable consumption in children and adolescents: a systematic review. Public Health Nutr 12, 267-283.

17. Hanson NI, Neumark-Sztainer D, Eisenberg ME, et al. (2005) Associations between parental report of the home food environment and adolescent intakes of fruits, vegetables and dairy foods. Public Health Nutr 8, 77-85.

18. Houldcroft L, Haycraft E \& Farrow C (2014) Peer and friend influences on children's eating. Soc Dev 23, 19-40.

19. Vereecken CA, Van Damme W \& Maes L (2005) Measuring attitudes, self-efficacy, and social and environmental influences on fruit and vegetable consumption of 11- and 12-yearold children: reliability and validity. J Am Diet Assoc 105, 257-261.

20. De Bourdeaudhuij I \& Van Oost P (2000) Personal and family determinants of dietary behaviour in adolescents and their parents. Psychol Health 15, 751-770.

21. Bruening M, Eisenberg M, MacLehose R, et al. (2012) Relationship between adolescents' and their friends' eating behaviors: breakfast, fruit, vegetable, whole-grain, and dairy intake. I Acad Nutr Diet 112, 1608-1613.

22. Pearson N, Ball K \& Crawford D (2011) Predictors of changes in adolescents' consumption of fruits, vegetables and energydense snacks. Br J Nutr 105, 795-803.

23. Kristjansdottir AG, Thorsdottir I, De Bourdeaudhuij I, et al. (2006) Determinants of fruit and vegetable intake among 11-year-old schoolchildren in a country of traditionally low fruit and vegetable consumption. Int J Behav Nutr Phys Act 3, 41 .

24. Sandvik C, De Bourdeaudhuij I, Due P, et al. (2005) Personal, social and environmental factors regarding fruit and vegetable intake among schoolchildren in nine European countries. Ann Nutr Metab 49, 255-266.

25. Bere E, Brug J \& Klepp K (2007) Why do boys eat less F\&V than girls? Public Health Nutr 11, 321-325.

26. Glasson C, Chapman K \& James E (2011) Fruit and vegetables should be targeted separately in health promotion programmes: differences in consumption levels, barriers, knowledge and stages of readiness for change. Public Health Nutr 14, 694-701.

27. Reinaerts E, de Nooijer J, Candel M, et al. (2007) Explaining school children's fruit and vegetable consumption: the contributions of availability, accessibility, exposure, parental consumption and habit in addition to psychosocial factors. Appetite 48, 248-258.

28. Haraldsdóttir J, Thorsdottir I, Vaz de Almeida MD, et al. (2005) Validity and reproducibility of a precoded questionnaire to assess fruit and vegetable intake in European 11- to 12-year-old schoolchildren. Ann Nutr Metab 49, 221-227.

29. De Bourdeaudhuij I, Klepp K, Due P, et al. (2005) Reliability and validity of a questionnaire to measure personal, social and environmental correlates of fruit and vegetable intake in 10-11-year-old children in five European countries. Public Health Nutr 8, 189-200.

30. Clayton D \& Hills M (1993) Statistical Models in Epidemiology. Oxford: Oxford University Press.

31. Muthén L \& Muthén B (1998-2012) Mplus User's Guide. Los Angeles, CA: Muthén \& Muthén.

32. Ray C \& Roos E (2012) Family characteristics predicting favourable changes in 10 and 11-year-old children's lifestylerelated health behaviours during an 18-month follow-up. Appetite 58, 326-332.

33. Talvia S, Lagström H, Räsänen M, et al. (2004) Calorie (energy) and nutrient intakes up to the age of 10 years in the Special Turku Coronary Risk Factor Intervention Project. Arch Pediatr Adolesc Med 158, 41-47. 
34. Kristjansdottir AG, De Bourdeaudhuij I, Klepp KI, et al. (2009) Children's and parents' perceptions of the determinants of children's fruit and vegetable intake in low-intake population. Public Health Nutr 12, 1224-1233.

35. Bjelland M, Lien N, Grydeland M, et al. (2011) Intakes and perceived home availability of sugar-sweetened beverages, fruit and vegetables as reported by mothers, fathers and adolescents in the HEIA (HEalth In Adolescents) study. Public Health Nutr 14, 2156-2165.

36. Pedersen S, Grønhøj A \& Thøgersen J (2015) Following family or friends. Social norms in adolescent healthy eating. Appetite 86, 54-60.
37. Stok FM, de Ridder DT, de Vet E, et al. (2014) Don't tell me what I should do, but what others do: the influence of descriptive and injunctive peer norms on fruit consumption in adolescents. Br J Health Psychol 19, 52-64.

38. Horne P, Tapper K, Lowe C, et al. (2004) Increasing children's fruit and vegetable consumption: a peer-modelling and rewards-based intervention. Eur J Clin Nutr 58, 1649-1660.

39. Bere E \& Klepp K-I (2003) Reliability of parental and self-reported determinants of fruit and vegetable intake among 6th graders. Public Health Nutr 7, 353-356.

40. Draxten M, Fulkerson JA, Friend S, et al. (2014) Parental role modeling of fruits and vegetables at meals and snacks is associated with children's adequate consumption. Appetite 78, 1-7. 